\title{
Quantitative Stereological Estimations of Structural Patterns of the Glandular Tree in Benign Hyperplasia of Prostate
}

\author{
Luis Santamaría ${ }^{*}$, Ildefonso Ingelmo², Fernando Teba ${ }^{3}$, Almudena Coloma ${ }^{4}$, \\ Laura Martínez ${ }^{4}$ \\ ${ }^{1}$ Department of Anatomy, Histology, and Neuroscience, School of Medicine, Autonomous University of \\ Madrid, Madrid, Spain \\ ${ }^{2}$ Department of Anesthesiology, Hospital Ramón y Cajal, Madrid, Spain \\ ${ }^{3}$ Department of Surgery (Urology), Hospital de La Princesa, School of Medicine, Autonomous University of \\ Madrid, Madrid, Spain \\ ${ }^{4}$ Department of Surgery (Urology), Hospital del Henares, Madrid, Spain \\ Email: "Iuis.santamaria@uam.es
}

Received 22 April 2016; accepted 26 June 2016; published 29 June 2016

Copyright (C) 2016 by authors and Scientific Research Publishing Inc. This work is licensed under the Creative Commons Attribution International License (CC BY). http://creativecommons.org/licenses/by/4.0/

\section{(c) (i) Open Access}

\section{Abstract}

Background: Benign prostate hyperplasia (BPH) is the most common benign disease of human prostate. Currently BPH is associated with unregulated proliferation of connective tissue and glandular epithelium within the prostatic transition zone, and it has been described as relevant characteristic of $\mathrm{BPH}$ - the increase of the total number of cells, and not only an increase in cell size. To date, there are few studies on the quantitative morphology of glandular tree of BPH compared with normal prostate. The scarce investigations about this particular suggest that the glandular tree branches and expands as the hyperplastic transformation occurs in the prostate. Methods: To verify if this gland expansion and branching was similar to that occurs in the normal prostate, this study deals with the estimation of several stereological parameters as: labeling index for the proliferating cell nuclear antigen to quantify the rate of proliferation of prostate epithelium, average thickness of glandular epithelium, fraction of the volume occupied by the epithelium relative to the total prostate volume, connectivity density of prostate glands, to quantify the branching of prostate glands, and the average volume and the volume-weighted mean glandular volume of prostate acini to assess the mean size of the prostate acini and its variability. Results: All these estimates have been performed in prostate specific antigen immunostained sections from prostates of young men (controls) and in adenomectomy specimens from the adenofibromiomatous variety of BPH. Conclusion: We conclude that the epithelial proliferation is not the only factor intervening in the development of BPH. In addition, a more prolonged survival of epithelial

*Corresponding author.

How to cite this paper: Santamaría, L., Ingelmo, I., Teba, F., Coloma, A. and Martínez, L. (2016) Quantitative Stereological Estimations of Structural Patterns of the Glandular Tree in Benign Hyperplasia of Prostate. Open Journal of Pathology, 6, 122-133. http://dx.doi.org/10.4236/ojpathology.2016.63015 
population, together with some degree of hypertrophy of acini expressed by the increase of volume fraction and thickness of acinar epithelium, is relevant in order to the growth and expansion of the BPH glandular tree that shows more abundant and heterogeneous acinar sprouts than in normal prostate.

\section{Keywords}

Prostate, Hyperplasia, Cell Proliferation, Gland Volume, Variability on Size

\section{Introduction}

Benign prostate hyperplasia (BPH) is the most common benign disease of human prostate [1] [2]. It produces urological symptoms in 54\% of men between 60 and 70 years. The BPH detection by microscopic examination at autopsy is greater than the clinic detection of symptoms (about $70 \%$ of men in the same age range) [3]. It represents approximately $50 \%$ of the medical consultations made by urological disease [4] [5].

BPH has two phases: pathologic and clinical. The first phase has in turn two stages: microscopic, with the presence of small foci of prostatic hyperplasia, and macroscopic, with evidence of adenomas. Approximately $80 \%$ of men over 40 years developed a microscopic $\mathrm{BPH}$, but only half of these evolved to a macroscopic BPH in an average time of five years [6] [7].

The prostate grows from birth to puberty to reach between 20 and 30 years of age reaching an average weight of $20 \mathrm{~g}$. From here to the 90 years its growth rate decreases. From the fifth decade of life, with the development of histologically identifiable BPH, increased prostate weight occurs reaching an average weight of $33 \mathrm{~g}$ [8].

Prostatic stromal cells play an important role in the pathogenesis of BPH. The first histological changes include microscopic nodules in the stroma surrounding the periurethral glands. The acinar hyperplasia begins around these small nodules and will increase over the years, to produce nodules up to several centimeters in diameter whose histology may show a predominance of epithelial elements, stromal or a mixed pattern. Depending on what elements predominate, BPH can be classified in five varieties: stromal, fibromuscular, muscular, fibroadenomatous and adenofibromiomatous (the most common) [8]. BPH affects mostly the transition zone and the periurethral area of the prostate [9] [10].

Although the pathogenesis of BPH is unclear, it is known to be multifactorial, being necessary the presence of two factors for prostate growth occurs: the androgen stimulus and age [4]. Different theories have been proposed, based on histological, and hormonal age-related changes, but, currently, not a single explanation is accepted [11]. Androgens possibly act as initiators' stromal hyperplasia, which in turn induce epithelial hyperplasia [3] [12]. It has been also suggested that neuroendocrine cells are involved in the genesis of $\mathrm{BPH}$, perhaps enacting some mediation between stroma and epithelium.

Currently BPH is associated with unregulated proliferation of connective tissue, smooth muscle and glandular epithelium within the prostatic transition zone, and it has been described as relevant characteristic of BPH-the increase of the total number of cells, and not only an increase in cell size [13]. [14] has shown that early periurethral nodules have a stromal structure, while the early nodules in the transition zone represent the proliferation of the glandular tissue. Glandular nodules rise from the newly formed small ducts, arising as buds on existing ducts; these ducts grow and branch out, creating an entirely new ductal system within the nodule. Histologically, BPH is characterized by progressive hyperplasia of glandular and stromal tissues around the urethra with excessive nodular growth localized to the points where ejaculatory ducts enter into the transitional or periurethral zones of the prostate [15].

The cytological findings in BPH are generally of little relevance, and rather unspecific, as alterations including basal cell hyperplasia, increased stromal mass (particularly the amount of smooth muscle cells), enhanced extracellular matrix deposition, reduced elastic tissue, more infiltrating lymphocytes around ducts, acinar hypertrophy and more luminal corpora amylacea and calcifications in the form of prostatic calculi [16].

Although some increase of cell proliferation has been detected in the glandular epithelium of BPH in comparison with normal prostate [17], the proliferating rate was significantly lower than in prostate cancer [18]. In both normal and BPH glands the proliferative compartment is located in the basal cell layer. Compared to normal and 
hyperplastic conditions, severe proliferative abnormalities were detected in high-grade prostate intraepithelial neoplasia, as documented by the extension of the proliferative compartment up to the luminal border [19].

To date, there have been no studies on the quantitative morphology of glandular tree of BPH compared with normal prostate. The only mention of this particular has been noted by [20], indicating that the histologic hallmark of BPH is the expansile nodule, produced by the budding and branching of newly formed duct-acinar structures. Suggesting that the glandular tree branches and expands as the hyperplastic transformation occurs in the prostate.

To verify if this gland expansion and branching was similar to that occurs in the growth of normal prostate, the present work deals with the estimation of several stereological parameters which can shed some light on the structure and distribution of prostatic glandular tree and its possible changes in $\mathrm{BPH}$.

Briefly, the parameters investigated were:

1) Labeling index for the proliferating cell nuclear antigen (PCNA) to quantify the rate of proliferation of prostate epithelium.

2) Average thickness of glandular epithelium.

3) Fraction of the volume occupied by the epithelium relative to the total prostate volume.

4) Connectivity density of prostate glands, to quantify the branching of prostate glands.

5) The average volume and the volume-weighted mean glandular volume of prostate acini to assess the mean size of the prostate acini and its variability.

All these estimates have been performed in prostates from young men (controls) and in adenomectomy specimens from the adenofibromiomatous variety of $\mathrm{BPH}$.

\section{Methods}

Twenty prostate specimens were collected at La Princesa Hospital (Madrid, Spain), 10 were from adults, (CTR group), age (mean $\pm \mathrm{SD}$ ): $45 \pm 7$; range: 30 - 47 years, all these specimens were of healthy subjects, without endocrine or reproductive pathology, deceased in traffic accidents, and eligible as donors for transplant, the age of the subjects of CTR group was in the range indicated to avoid any histological changes of subclinical BPH, relatively frequents in subjects older than 50 years. The other 10 were surgical specimens (adenomectomies) from patients diagnosed of the adenofibromiomatous type of benign prostatic hyperplasia, (BPH group), age (mean \pm SD): $75 \pm 10$, range: 65 - 85 years. All the ethical requirements were accomplished in order to obtain the prostatic tissue either at the moment of the multiorganic extraction for transplant (CTR group) or at the surgery (BPH group). Immediately after extraction, the specimens were fixed during a week in 10\% paraformaldehyde in PBS, pH 7.4 (Probus, Barcelona, Spain).

After fixation, the specimens from the two groups were thoroughly sectioned into 2-mm-thick slices, performed by isotropic uniform random sampling (IUR sections) in order to preserve the isotropy of the tissue [21].

All the specimens were processed for paraffin embedding. The paraffin blocks were exhaustively sectioned. A total of 20 sections were performed on each block: Ten 5- $\mu$ m-thick sections for immunohistochemistry and routine haematoxylin-eosine, and other ten 15 - $\mu$ m-thick sections for quantitative studies.

Two immunostainings were performed: i) for visualization of proliferating cell nuclear antigen (PCNA) in order to evidence the epithelial proliferating cells [22], and ii) for detection of the prostatic specific antigen (PSA), to visualize the prostate acini [23].

At least five randomly selected slides per specimen were immunostained for each antigen in CTR and BPH groups. Deparaffinized and rehydrated tissue sections were treated for 30 min with hydrogen peroxide $0.3 \%$ in phosphate-buffered saline (PBS) pH 7.4, to block endogenous peroxidase. To detect PCNA immunoreactivity, sections were incubated with a monoclonal anti-PCNA antibody (Biomeda, Foster City, Ca, USA) diluted at 1:400. To show PSA immunoreactivity, sections were incubated with a monoclonal anti-PSA antibody (Santa Cruz Biotechnology, Santa Cruz, Ca, USA) diluted at 1:50. Pretreatment of sections by heat in citrate buffer pH 6.0 (using a pressure cooker) [24] was performed to enhance immunostaining for both antibodies.

The primary antisera were diluted in PBS pH 7.4 containing $1 \%$ bovine serum albumin (BSA) (Sigma, St Louis, USA) plus $0.1 \%$ sodium azide (Merck, Darmstad, Germany). The incubation with primary antisera was overnight at $4^{\circ} \mathrm{C}$. The second antibodies employed were a biotin-caproyl-anti-rabbit immunoglobulin (Biomeda, Foster City, CA, USA). The second antibodies were diluted at 1/400 in PBS containing 1\% BSA without sodium azide, and incubated for $30 \mathrm{~min}$ at room temperature. Thereafter, sections were incubated with a streptavi- 
din-biotin-peroxidase complex (Biomeda). The immunostaining reaction product was developed using $0.1 \mathrm{~g}$ diaminobenzidine (DAB) (Sigma) in $200 \mathrm{~mL}$ of PBS, plus $40 \mu \mathrm{L}$ hydrogen peroxide. After immunoreaction nuclear counter-staining with haematoxylin was performed in all the sections immunostained for PCNA, and in some sections immunostained for PSA. No nuclear counter-staining was performed on the 15- $\mu$ m-thick sections PSA immunostained that were then employed for quantitative purposes. All slides were dehydrated in ethanol, and mounted in a synthetic resin (Depex, Serva, Heidelberg, Germany). The specificity of the immunohistochemical procedures was checked by incubation of sections with nonimmune serum instead of the primary antibody.

\section{Quantitative Methods}

\section{i) Estimation of the proliferative index of epithelium}

In the two groups, the numerical density $\left(N_{V}\right)$ of epithelial cells (cell number per unit of volume of reference space) was estimated for both PCNA immunoreactive ( $N_{V}$ PCNA positive) and PCNA negative nuclei $\left(N_{V}\right.$ PCNA negative), employing the optical disector, an unbiased stereological method [25], using the Cast-Grid programme (Stereology Software Package, Silkeborg, Denmark). The space of reference employed for estimating the numeric densities was the occupied by the epithelium, then, only was registered the volume associated to the frames with its upper-right corner hitting epithelial tissue. The total $N_{V}$ of epithelial cells was calculated as $N_{V}$ total $=N_{V}$ (PCNA negative + PCNA positive), then, the labeling index to PCNA (LIpcna) for epithelial cells was expressed as the ratio $N_{V}$ PCNA positive/ $N_{V}$ total.

\section{ii) Quantitative morphology of prostate acini}

Three parameters have been employed to quantitate the acinar pattern: The volume fraction of epithelium $\left(\mathrm{V}_{\mathrm{V}}\right.$ ep), the average thickness of epithelial lining (Th ep), and the connectivity density of the acinar tree $\left(\mathrm{Con}_{V} \mathrm{gl}\right)$.

The sampling protocol for the estimate of these parameters is described in detail: For all the specimens in each group of study, three sections immunostained to PSA were randomly sampled. Every section was scanned at a final magnification of $\times 100$, to select an average of 5 microscopical fields per section that were chosen by systematic random sampling [26]. For each selected field, five images evenly distributed along the $\mathrm{Z}$ axis of the section were captured, being the distance between each couple of images of $2 \mu \mathrm{m}$. The capturing of images was made using a colour digital camera DP 70 (Olympus Corporation of the Americas, PA, USA) with a resolution of 12.5 mega-pixels, attached to an Olympus microscope fitted with a motorised stage controlled by the stereological software Cast-Grid (Stereology Software Package, Silkeborg, Denmark). This program controls the XY displacement of the microscope stage and allows the selection of fields to be studied by random systematic sampling after the input of an appropriate sampling fraction [27]. The result was a series of images from the two groups, sized $512 \times 680$ pixels. The final magnification $(\times 100)$ was such that 512 pixels represented $653 \mu \mathrm{m}$.

Subsequently, the images were processed using the public domain Java image processing program, Image $\mathrm{J}$ (version 1.48), developed at the US National Institutes of Health and available on the Internet at http://imagej.nih.gov/ij/index.html [28]. For each set of images obtained in each sampled field, a stack of a thickness of $10 \mu \mathrm{m}$ was constructed. Afterwards, for estimation of $\mathrm{V}_{\mathrm{V}}$ ep, and Th ep, every stack was binarised, and the PSA immunostained cytoplasm was shown as black and the reference space (glandular lumina, stroma, etc.) as white (Figures 1(a)-(d)). Then, for estimation of $\mathrm{Con}_{\mathrm{V}}$ gl, the binarised stack was subsequently processed by several stages of pixel dilation and hole filling to transform the binarised acini (epithelia plus lumina) in compact contours where the acini were shown as black and the stroma as white (Figure 1(e), Figure $1(f))$.

The quantitative parameters studied were estimated onto these stacks of binarised images, employing a plugin that runs in the Image J software [29], briefly:

a) Epithelial volume fraction ( $V_{V}$ ep) is the volume of PSA immunoreactive epithelium per unit of volume of the reference space (acini plus stroma), $\mathrm{V}_{\mathrm{V}}$ ep is simply the number of foreground (epithelium) voxels divided by the total number of voxels in the image.

b) Average thickness of epithelial lining (Th ep). The plugin employed defines the thickness at a point as the diameter of the greatest sphere that fits within the structure (epithelial lining) and which contains the point [30]. The plugin calculates the average of the epithelial thickness (Th ep) directly from pixel values in the resulting thickness map. It assumes that PSA immunostained epithelium is the foreground of the binarised image, the results are shown in $\mu \mathrm{m}$. 


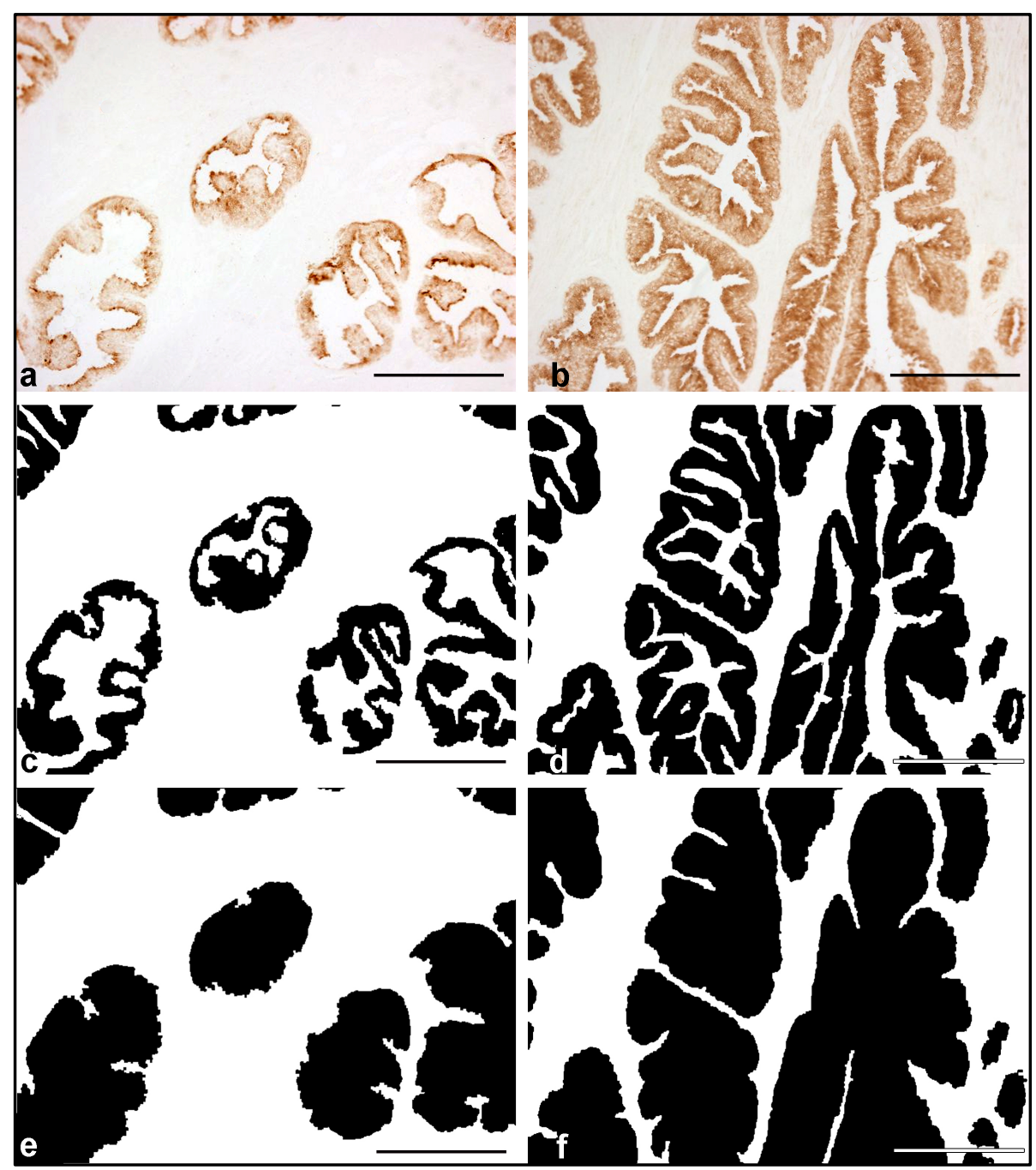

Figure 1. In (a) and (b) the images shown are from a specimen of CTR group and a case of $\mathrm{BPH}$ group respectively, immunostained to PSA. The same binarised images are depicted in (c) and (d), the PSA immunoreactive cytoplasm is in black, and the space of reference (acinar lumina and stroma) in white. These binarised pictures, processed to obtain the contours of acinar profiles (epithelium plus lumina, in black) are shown in (e) and (f). The scale bars represent $190 \mu \mathrm{m}$.

c) Connectivity density of the acinar tree (Con $\mathrm{V}$ gland). The number of connected structures in a network can be determined by calculating the Euler characteristic [31]. As other structures, like lung alveoli or cancellous bone [32] [33], prostate glandular tree is one such network, and its connectivity density $\left(\mathrm{Con}_{\mathrm{V}}\right.$ gl), i.e. number of acini connected per unit of volume of reference space, can by calculated by dividing the connectivity estimate by the volume of the stack sampled. The algorithm in the plugin employed uses voxel neighborhoods to calculate the Euler characteristic of the volume sampled in the binarised stack, and adjusts this to give the contribution of this volume to the connectivity of the structure it was chosen from. The amount of connected structures (acini) gives an idea of the complexity of glandular branching.

iii) Estimations of size of the prostate acini

Two methods have been performed in order to measure the mean volume of the acini, first was estimated the average volume of glands that were sampled independently of their size [34], i.e. the not weighted or numeric distribution of size $\left(V_{N} g l\right)$. Then was obtained a volume-weighted mean glandular volume [35], that provides a weighted distribution of size $\left(V_{V} g l\right)$ that gives information about the variability of size population [34].

The procedures were as follows: 
a) The nucleator, an unbiased stereological estimator [36], was applied to estimate the mean glandular volume $\left(V_{N} g l\right)$ for the prostate acini stained with HE from both groups of study. Briefly: The acini eligible for measuring were sampled after Sterio rule [37] using optical disectors at a final magnification of $\times 40$. Their center of mass were determined and employed as the reference point. Through this point, the software generates two isotropically oriented segments hitting four points from the boundary of the gland. The program then employs the following formula to estimate the volume: $V_{N}=4 \pi / 3 \cdot l_{n}^{3}$, where $V_{N}$ is the neuronal volume, and $l_{n}^{3}$ is the mean cubed length of the segments intercepting the boundary of the acini.

b) The average volume-weighted mean glandular volume $\left(V_{V} g l\right)$ was performed using the point sampled intercept method [34], that estimates the volume, giving greater chance of volume estimation to structures of greater size.

The program used to evaluate $V_{V} g l$ enables the generation of random test-lines directions that were superimposed onto the microscope images. The gland intercepts can be measured along these test lines. The length of gland intercepts $\left(l_{0}\right)$ was processed to obtain $\pi / 3 \cdot l_{0}^{3}$, an unbiased estimate of $V_{V} g l$ independent of acinar shape, which, because of point sampling, emphasizes larger acini rather than smaller ones. In addition, estimates of $V_{V}$ gl combine information about the three-dimensional glandular size with knowledge of variability of glandular size [38].

All the measurements from $V_{N} g l$ and $V_{V} g l$ were carried out in each group of study onto three sections per specimen stained with haematoxylin-eosine and randomly sampled. The fields selected were visualized using an Olympus microscope at a final magnification of $\times 40$. The software employed by measurements was the Cast-Grid.

c) Estimation of the second moment of the $V_{N} g l$ distribution: As estimates of $V_{N} g l$ and $V_{V} g l$ were made on the same population of glands, the coefficient of variation of the acinar volumes in the $V_{N} g l$ distribution [39] is given by

$$
C V_{N}(V) g l=\sqrt{\frac{V_{V} g l}{V_{N} g l}-1}
$$

where $C V_{N}(V) g l$ is the coefficient of variation of the acinar volume in the $V_{N} g l$ distribution that is a measure of the variability in size of acini.

\section{iv) Statistical analysis}

The parameters measured were expressed as mean \pm CI (confidence intervals at 95\%). Comparisons between the means from CTR and BPH groups were performed by a Student $t$ test $(p<0.05)$.

\section{Results}

\subsection{Immunohistochemistry}

Immunoreactivity for PCNA has been detected in some nuclei from basal cells of acinar epithelium in both control and BPH specimens. Not relevant differences were observed in intensity and distribution of PCNA immunoreactive nuclei in both groups of study (Figure 2). The cytoplasm of epithelial cells from normal (CTR group) and hyperplastic (BPH group) acini showed intense immunoreaction to PSA (Figure 3). No immunostaining to PSA was detected in other locations of normal and BPH prostates.

\subsection{Quantitative Results}

The LI pcna did not experiment significant changes when comparing CTR and BPH groups (Figure 4(a)). However, the epithelial thickness (Th ep) and the volume fraction of prostate occupied by epithelium ( $V_{V}$ ep) were significantly increased in BPH group in comparison with CTR group (Figure 4(b), Figure 4(c)). Similarly, connectivity density $\left(\mathrm{Con}_{V} \mathrm{gl}\right)$ increases significantly in CTR group compared to the BPH group (Figure 4(d)).

The average volume of the prostate acini $\left(V_{N} g l\right)$ does not change significantly in BPH in comparison with CTR groups (Figure 5(a)), whereas the volume-weighted mean glandular volume $\left(V_{V} g l\right)$ and the coefficient of variation of the acinar volume $-C V_{N}(V)$ gl- were significantly increased in BPH specimens (Figure 5(b), Figure 5(c)).

\section{Discussion}

In the present study, it has been noted that in BPH there is no significant increase of cell proliferation measured 


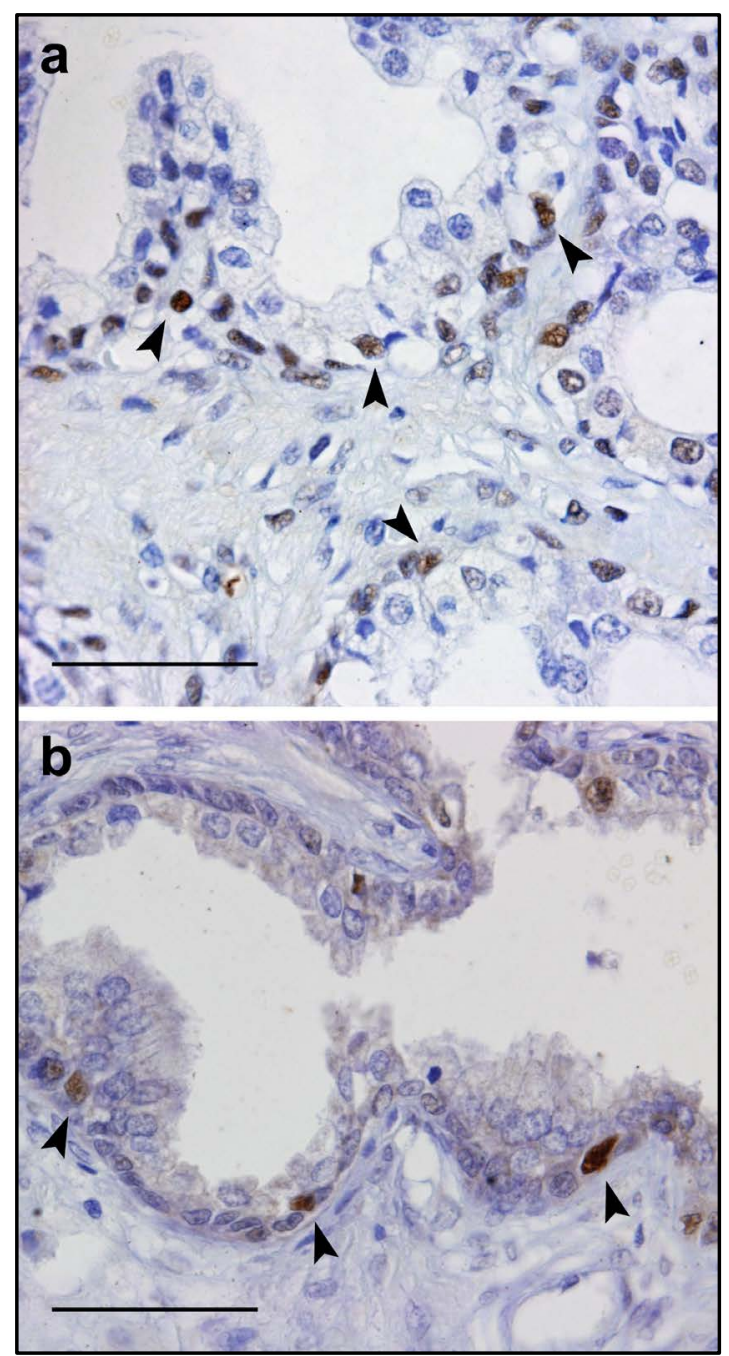

Figure 2. Sections immunostained to PCNA, from a specimen of CTR group (a) and from a case of BPH group (b). Some nuclei of epithelial basal cells are immunoreactive (arrowheads). No immunostaining for PCNA is viewed in the columnar cells of epithelium in both images. The scale bars represent $50 \mu \mathrm{m}$.

by PCNA labeling index. This seems in contrast to that described by other authors [18] [19] suggesting an increase in cell proliferation rate in BPH compared to normal prostate. This discrepancy may be due to that the BPH specimens included in the present study are in an advanced stage of hyperplastic growth, where more than a high cell proliferation, increased apoptosis occurs, keeping the size of the epithelial population, since it is known that prostatic hyperplasia shows minor degree of apoptosis that normal prostate, being an initial growth imbalance in favor of cell proliferation that might promote prostatic hyperplasia [17].

The end point of this shifting towards the cell proliferation is the increase of epithelium that was reflected in the enlargement of the volume fraction of prostate gland occupied by the epithelium as was described in this study. However, other authors [40], detect an increase of volume fraction of stroma instead of acini, this discrepancy might be due to the type of BPH studied. The augmentation of the epithelial compartment would be preferently found in the adenofibromiomatous type of BPH [8] that was the variety of BPH studied in this work. Also can be detected an increase of stroma-epithelium ratio in symptomatic BPH in comparison with asymptomatic BPH [41].

The increase of thickness of epithelial lining might be in relation to some hypertrophy at the cellular level that is also present [42]. 


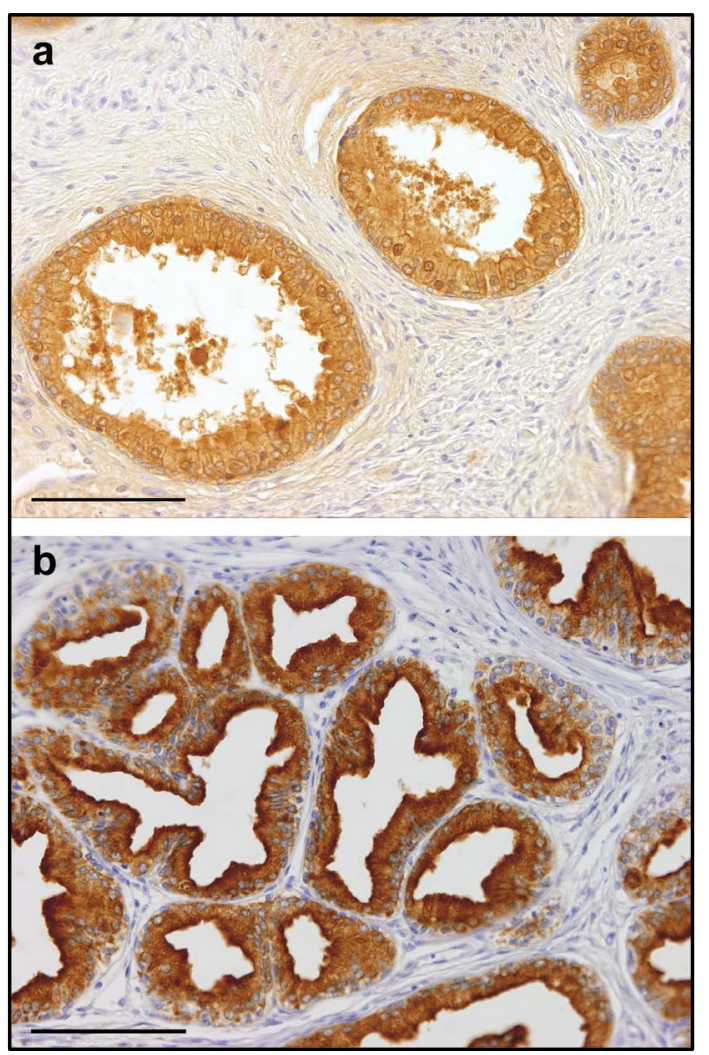

Figure 3. Sections immunostained to PSA: in (a) image from a CTR case, in (b) an image from a BPH case. The cell nuclei were counterstained with haematoxylin. The scale bars represent $100 \mu \mathrm{m}$.
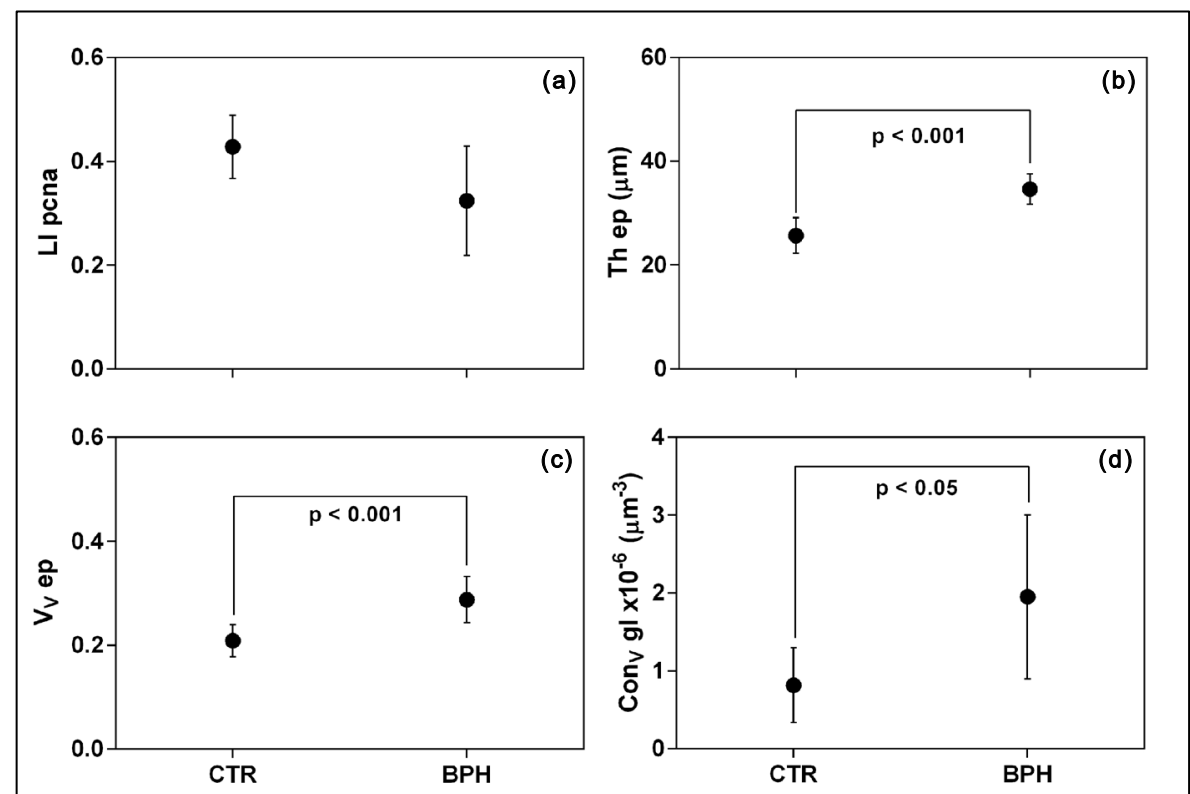

Figure 4. Mean \pm CI (confidence intervals at 95\%) for: (a) Epithelial labeling index for PCNA (LI pcna), (b) Average thickness of epithelium (Th ep), (c) Volume fraction of PSA immunoreactive epithelium ( $\mathrm{V}_{\mathrm{V}}$ ep), and (d) Density of connectivity of prostate glands (Con $\left.\mathrm{V}_{\mathrm{V}} \mathrm{gl}\right)$, in control (CTR) and hyperplastic (BPH) groups. The means with significant differences between them are connected by zig-zag lines, indicating the correspondent $\mathrm{p}$ values. 


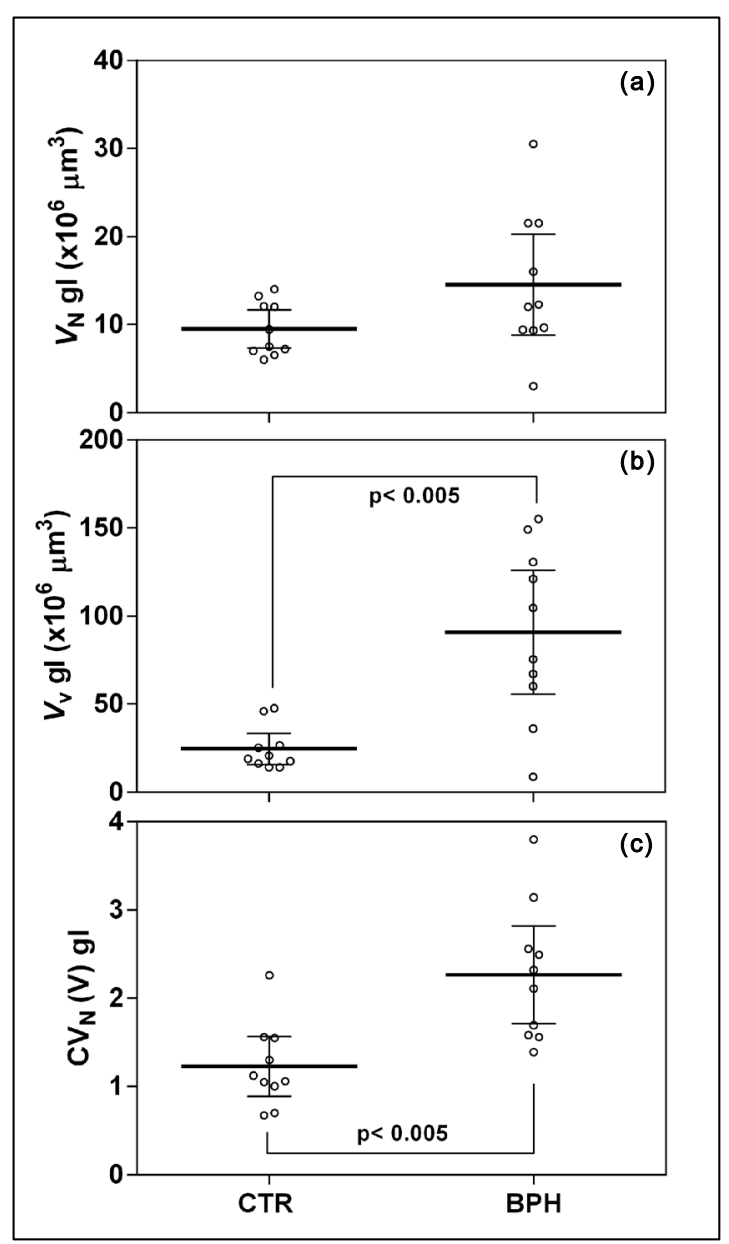

Figure 5. Scatter plots expressing individual values (void circles) plus mean \pm CI (confidence intervals at 95\%) for: (a) numeric distribution of gland size $\left(V_{N} g l\right)$, (b) average volume-weighted mean glandular volume $\left(V_{V} g l\right)$, and (c) coefficient of variation of the acinar volume in the $V_{\mathrm{N}}$ gl distribution $\left[C V_{N}(V) \mathrm{gl}\right]$. The means with significant differences between them are connected by zig-zag lines, indicating the correspondent $\mathrm{p}$ values.

The connectivity density from acini in BPH was significantly higher than in control prostate.

This parameter is related with the number of patterns interconnected in a branching structure [31] as occurs for the prostate glands. The increase of $\mathrm{Con}_{\mathrm{V}}$ gl suggest that in $\mathrm{BPH}$, the glandular tree branches and expands as the hyperplastic transformation occurs in the prostate [20]. It is interesting to note, that the average acinar size estimated by $V_{N}$ does not change in BPH specimens comparing with control cases. Therefore, the increment of epithelial volume fraction correlates more with the increase of the number of acinar buds that with an increasing of mean volume of such elements.

The average volume-weighted mean volume is a simple and efficient method of estimating the size of particles, and has been revealed to be an important prognostic factor in a number of histopathological studies of melanoma [43], prostate cancer [35] [44], ovarian cancer [45] and others. All these studies deals with the size of cell nuclei, but to our knowledge the volume-weighted volume has not been employed to evaluate the variability on size of prostate acini.

The $V_{V} g l$ of the acini was significantly increased in BPH cases in comparison with CTR group. This finding is interesting because estimates of $V_{V} g l$ combine information about the three-dimensional glandular size with knowledge of variability of glandular size [38]. In fact, the coefficient of variation of acinar volumes in the $V_{N} g l$ distribution was also significantly greater in BPH than in CTR. Therefore, the size of the acini in BPH is more 
heterogeneous than the acini controls. That may mean that the growth of acinar buds in hyperplastic prostate is irregular and not fully comparable to what happens in the not hyperplastic gland.

\section{Conclusion}

This study sheds some new light on the pathogenesis of BPH, from the point of view of the growing pattern of the hyperplastic glandular tree. We can conclude that the epithelial cell proliferation is not the only factor intervening in the development of $\mathrm{BPH}$, at least, in the stage, or for the type of hyperplasia studied in this work. In addition, a more prolonged survival of epithelial population, together with some degree of hypertrophy of acini expressed by the increase of volume fraction and thickness of acinar epithelium, is relevant in order to the growth and expansion of the BPH glandular tree that shows more abundant and heterogeneous acinar sprouts than in normal prostate.

\section{References}

[1] Barry, M.J. (1990) Epidemiology and Natural History of Benign Prostatic Hyperplasia. Urologic Clinics of North America, 17, 495-507.

[2] Kirby, R.S. (1992) The Clinical Assessment of Benign Prostatic Hyperplasia. Cancer, 70, 284-290. http://dx.doi.org/10.1002/1097-0142(19920701)70:1+<284::AID-CNCR2820701316>3.0.CO;2-\#

[3] Birkhoff, J.D. (1983) Natural History of Benign Prostatic Hypertrophy. In: Hinman, F., Ed., Benign Prostatic Hypertrophy, Springer Verlag, New York, 5-14. http://dx.doi.org/10.1007/978-1-4612-5476-8_1

[4] Burgos Rodríguez, R. and Chicharro Molero, J.A. (1993) Hiperplasia Benigna de Próstata. In: Jiménez Cruz, J.F. and Rioja Sanz, L.A., Eds., Tratado de urología. J.R.Prous, Barcelona, 1035-1050.

[5] Isaacs, J.T. (1994) Etiology of Benign Prostatic Hyperplasia. European Urology, 25, 6-9.

[6] Oesterling, J.E. (1991) The Origin and Development of Benign Prostatic Hyperplasia. An Age-Dependent Process. Journal of Andrology, 12, 348-355.

[7] Fitzpatrick, J.M. (2006) The Natural History of Benign Prostatic Hyperplasia. BJU International, 97, 3-6. http://dx.doi.org/10.1111/j.1464-410X.2006.06097.x

[8] Berry, S.J., Coffey, D.S., Walsh, P.C. and Ewing, L.L. (1984) The Development of Human Benign Prostatic Hyperplasia with Age. Journal of Urology, 132, 474-479.

[9] Algaba, F. (1993) Bases morfológicas del desarrollo de la hiperplasia prostática. Patología, 26, 113-119.

[10] Di Silverio, F., D’Eramo, G., Flammia, G.P., Caponera, M., Frascaro, E., Buscarini, M., et al. (1993) Pathology of BPH. Minerva Urologica e Nefrologica, 45, 135-142.

[11] Partin, A.W., Oesterling, J.E., Epstein, J.I., Horton, R. and Walsh, P.C. (1991) Influence of Age and Endocrine Factors on the Volume of Benign Prostatic Hyperplasia. Journal of Urology, 145, 405-409.

[12] Narayan, P. (1992) Neoplasias de la próstata. In: McAninch, J.W., Ed., Smith's General Urology, Appleton and Lange.

[13] Patel, N.D. and Parsons, J.K. (2014) Epidemiology and Etiology of Benign Prostatic Hyperplasia and Bladder Outlet Obstruction. Indian Journal of Urology, 30, 170-176. http://dx.doi.org/10.4103/0970-1591.126900

[14] McNeal, J.E. (1978) Origin and Evolution of Benign Prostatic Enlargement. Investigative Urology, 15, 340-345.

[15] Untergasser, G., Madersbacher, S. and Berger, P. (2005) Benign Prostatic Hyperplasia: Age-Related Tissue-Remodeling. Experimental Gerontology, 40, 121-128. http://dx.doi.org/10.1016/j.exger.2004.12.008

[16] Bostwick, D.G., Cooner, W.H., Denis, L., Jones, G.W., Scardino, P.T. and Murphy, G.P. (1992) The Association of Benign Prostatic Hyperplasia and Cancer of the Prostate. Cancer, 70, 291-301.

http://dx.doi.org/10.1002/1097-0142(19920701)70:1+<291::AID-CNCR2820701317>3.0.CO;2-4

[17] Kyprianou, N., Tu, H. and Jacobs, S.C. (1996) Apoptotic versus Proliferative Activities in Human Benign Prostatic Hyperplasia. Human Pathology, 27, 668-675. http://dx.doi.org/10.1016/S0046-8177(96)90396-2

[18] Zhong, W., Peng, J., He, H., Wu, D., Han, Z., Bi, X., et al. (2008) Ki-67 and PCNA Expression in Prostate Cancer and Benign Prostatic Hyperplasia. Clinical \& Investigative Medicine, 31, E8-E15.

[19] Bonkhoff, H., Stein, U. and Remberger, K. (1994) The Proliferative Function of Basal Cells in the Normal and Hyperplastic Human Prostate. The Prostate, 24, 114-118. http://dx.doi.org/10.1002/pros.2990240303

[20] McNeal, J.E. (2007) Prostate. In: Mills, S.E., Ed., Histology for Pathologists, Lippincott Williams \& Wilkins, Philadelphia, 923-942.

[21] Baddeley, A. and Vedel Jensen, E. (2005) Uniform and Isotropic Uniform Designs. In: Baddeley, A. and Vedel, J.E., 
Eds., Stereology for Statisticians, Chapman \& Hall, Boca Raton, 155-173.

[22] Arriazu, R., Pozuelo, J.M., Martin, R., Rodriguez, R. and Santamaria, L. (2005) Quantitative and Immunohistochemical Evaluation of PCNA, Androgen Receptors, Apoptosis, and Glutathione-S-Transferase P1 on Preneoplastic Changes Induced by Cadmium and Zinc Chloride in the Rat Ventral Prostate. The Prostate, 63, 347-357. http://dx.doi.org/10.1002/pros.20192

[23] Watt, K.W., Lee, P.J., M’Timkulu, T., Chan, W.P. and Loor, R. (1986) Human Prostate-Specific Antigen: Structural and Functional Similarity with Serine Proteases. Proceedings of the National Academy of Sciences of the United States of America, 83, 3166-3170. http://dx.doi.org/10.1073/pnas.83.10.3166

[24] Martin, J.J., Martin, R., Codesal, J., Fraile, B., Paniagua, R. and Santamaria, L. (2001) Cadmium Chloride-Induced Dysplastic Changes in the Ventral Rat Prostate: An Immunohistochemical and Quantitative Study. The Prostate, 46, 11-20. http://dx.doi.org/10.1002/1097-0045(200101)46:1<11::AID-PROS1003>3.0.CO;2-K

[25] Santamaria, L., Martin, R., Martin, J.J. and Alonso, L. (2002) Stereologic Estimation of the Number of Neuroendocrine Cells in Normal Human Prostate Detected by Immunohistochemistry. Applied Immunohistochemistry \& Molecular Morphology, 10, 275-281. http://dx.doi.org/10.1097/00129039-200209000-00016

[26] Howard, C.V. and Reed, M.G. (2005) Unbiased Stereology: Three-Dimensional Measurement in Microscopy. 2nd Edition, Bios Scientific Publishers, Oxford.

[27] Santamaria, L., Ingelmo, I., Ruiz, J. and Teba, F. (2011) Study of the Distribution of Microvessels in Normal and Pathologic Prostate Using an Information-Based Similarity Analysis. Journal of Microscopy, 243, 303-314. http://dx.doi.org/10.1111/j.1365-2818.2011.03508.x

[28] ImageJ (2006) Image Processing and Analysis in JAVA. http://rsb.info.nih.gov/nih-image

[29] Doube, M., Klosowski, M.M., Arganda-Carreras, I., Cordelieres, F.P., Dougherty, R.P., Jackson, J.S., et al. (2010) BoneJ: Free and Extensible Bone Image Analysis in ImageJ. Bone, 47, 1076-1079. http://dx.doi.org/10.1016/j.bone.2010.08.023

[30] Dougherty, R. and Kunzelmann, K. (2007) Computing Local Thickness of 3D Structures with ImageJ. Microscopy and Microanalysis, 13, 1678-1679. http://dx.doi.org/10.1017/S1431927607074430

[31] Toriwaki, J. and Yonekura, T. (2002) Euler Number and Connectivity Indexes of a Three Dimensional Digital Picture. Forma, 17, 183-209.

[32] Odgaard, A. and Gundersen, H.J. (1993) Quantification of Connectivity in Cancellous Bone, with Special Emphasis on 3-D Reconstructions. Bone, 14, 173-182. http://dx.doi.org/10.1016/8756-3282(93)90245-6

[33] Hyde, D.M., Tyler, N.K., Putney, L.F., Singh, P. and Gundersen, H.J.G. (2004) Total Number and Mean Size of Alveoli in Mammalian Lung Estimated Using Fractionator Sampling and Unbiased Estimates of the Euler Characteristic of Alveolar Openings. The Anatomical Record Part A: Discoveries in Molecular, Cellular, and Evolutionary Biology, 227A, 216-226. http://dx.doi.org/10.1002/ar.a.20012

[34] Sorensen, F.B. (1991) Stereological Estimation of the Mean and Variance of Nuclear Volume from Vertical Sections. Journal of Microscopy, 162, 203-229. http://dx.doi.org/10.1111/j.1365-2818.1991.tb03132.x

[35] Teba, F., Martin, R., Gomez, V., Herranz, L.M. and Santamaria, L. (2007) Cell Proliferation and Volume-Weighted Mean Nuclear Volume in High-Grade Pin and Adenocarcinoma, Compared with Normal Prostate. Image Analysis \& Stereology, 26, 93-99. http://dx.doi.org/10.5566/ias.v26.p93-99

[36] Howard, C.V. and Reed, M.G. (1998) Unbiased Stereology: Three-Dimensional Measurement in Microscopy. Garland science/BIOS Scientific Publishers, Oxford.

[37] Sterio, D.C. (1984) The Unbiased Estimation of Number and Sizes of Arbitrary Particles Using the Disector. Journal of Microscopy, 134, 127-136. http://dx.doi.org/10.1111/j.1365-2818.1984.tb02501.x

[38] Gundersen, H.J. (1988) The Nucleator. Journal of Microscopy, 151, 3-21. http://dx.doi.org/10.1111/j.1365-2818.1988.tb04609.x

[39] Gundersen, H.J. and Jensen, E.B. (1985) Stereological Estimation of the Volume-Weighted Mean Volume of Arbitrary Particles Observed on Random Sections. Journal of Microscopy, 138, 127-142. http://dx.doi.org/10.1111/j.1365-2818.1985.tb02607.x

[40] Bartsch, G. (1977) Stereology, a New Quantitative Morphological Approach to Study Prostatic Function and Disease. European Urology, 3, 85-95.

[41] Shapiro, E., Becich, M.J., Hartanto, V. and Lepor, H. (1992) The Relative Proportion of Stromal and Epithelial Hyperplasia Is Related to the Development of Symptomatic Benign Prostate Hyperplasia. Journal of Urology, 147, 12931297.

[42] Urology Online: German Textbook of Urology (2016). http://www.urology-textbook.com/benign-prostatic-hyperplasia.html 
[43] Sorensen, F.B. and Ottosen, P.D. (1991) Stereological Estimation of Nuclear Volume in Benign and Malignant Melanocytic Lesions of the Skin. Inter- and Intraobserver Variability of Malignancy Grading. American Journal of Dermatopathology, 13, 99-107. http://dx.doi.org/10.1097/00000372-199104000-00001

[44] Nielsen, K., Berild, G.H., Bruun, E., Jorgensen, P.E. and Weis, N. (1989) Stereological Estimation of Mean Nuclear Volume in Prostatic Cancer, the Reproducibility and the Possible Value of Estimations on Repeated Biopsies in the Course of Disease. Journal of Microscopy, 154, 63-69. http://dx.doi.org/10.1111/j.1365-2818.1989.tb00568.x

[45] Mogensen, O., Sorensen, F.B., Bichel, P. and Jakobsen, A. (1992) Nuclear Volume and Prognosis in Ovarian Cancer. International Journal of Gynecological Cancer, 2, 141-146. http://dx.doi.org/10.1046/j.1525-1438.1992.02030141.X

\section{Submit or recommend next manuscript to SCIRP and we will provide best service for you:}

Accepting pre-submission inquiries through Email, Facebook, Linkedin, Twitter, etc A wide selection of journals (inclusive of 9 subjects, more than 200 journals)

Providing a 24-hour high-quality service

User-friendly online submission system

Fair and swift peer-review system

Efficient typesetting and proofreading procedure

Display of the result of downloads and visits, as well as the number of cited articles

Maximum dissemination of your research work

Submit your manuscript at: http://papersubmission.scirp.org/ 